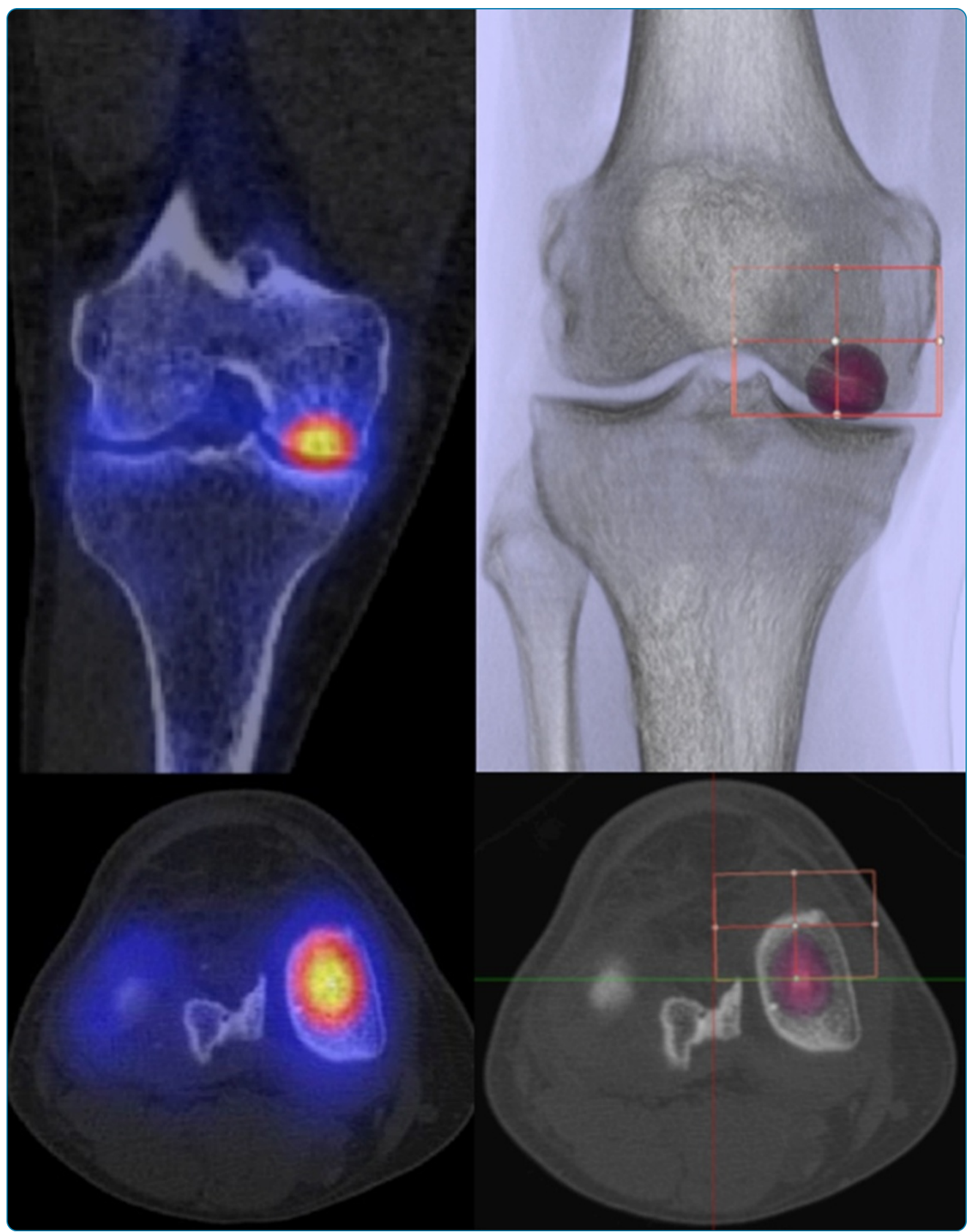

Assessment of the loading history of patients after high tibial osteotomy using SPECT/CT - a new diagnostic tool and algorithm

Mucha et al. 


\title{
Assessment of the loading history of patients after high tibial osteotomy using SPECT/CT - a new diagnostic tool and algorithm
}

\author{
Armin Mucha ${ }^{1}$, Milos Dordevic ${ }^{1}$, Enrique A Testa ${ }^{1}$, Helmut Rasch $^{2}$ and Michael T Hirschmann ${ }^{1 *}$
}

\begin{abstract}
Background: Single photon emission computerized tomography and conventional computerized tomography (SPECT/CT) tracer uptake has been shown to reflect the loading history of the tibiofemoral knee joint and correlate with the mechanical and anatomical alignment. It was our primary purpose to develop a novel standardized SPECT/CT algorithm for patients undergoing high tibial osteotomy, evaluate the inter- and intra-observer reliability $(\mathrm{OR})$, and assess the clinical applicability for follow-up of patients before and after high tibial osteotomy.

Methods: The localization scheme defines 9 femoral, 8 patellar, and 13 tibial zones to accurately map the examined tracer uptake volume in each anatomical area of interest. Maximum values for each area (mean \pm standard deviation, median, and range) of the localization scheme were recorded as well as normalized values for the intensity of SPECT/CT tracer uptake calculated. The inter- and intra-OR was assessed for SPECT/CT localization and tracer activity. Pre- and postoperative mechanical alignment was assessed in SPECT/CT using a custom-made specialized software. The median inter- and intra-observer differences of the measured mechanical alignment were calculated along with the inter- and intra-OR.
\end{abstract}

Results: The localization scheme showed near-perfect inter- and intra-OR (intra-class correlation coefficient $($ ICC) $>0.9$ ) for the measurement of tracer activity and localization in all anatomical regions. For measurements of mechanical alignment, there was a strong agreement between the two observers (an inter-OR of ICC $=0.99$ and an intra-OR of ICC $=0.98$ ).

Conclusions: The presented SPECT/CT algorithm is highly reliable and clinically feasible. Combined with mechanical alignment analysis, it provides the surgeon with helpful information about realignment effects of high tibial osteotomies (HTOs) and might help identify the optimal personalized degree of correction in HTO surgery.

Keywords: Knee, SPECT/CT, High tibial osteotomy, Diagnostics, Loading history, Pain, Realignment, Mechanical alignment

\section{Background}

Combined single photon emission computerized tomography and conventional computerized tomography (SPECT/CT) has yet proven useful as part of the diagnostic armamentarium in challenging orthopedic patients [1-7]. SPECT/CT tracer uptake is significantly correlated with the mechanical and anatomical alignment of the limb as well as with the degree of osteoarthritis [8].

\footnotetext{
* Correspondence: Michael.Hirschmann@unibas.ch

${ }^{1}$ Department of Orthopaedic Surgery and Traumatology, Kantonsspital

Baselland-Bruderholz, Bruderholz 4101, Switzerland

Full list of author information is available at the end of the article
}

It represents the loading history of the knee, and thus, overloading can be assessed at an early stage. Due to its unique characteristics, SPECT/CT promises information on the loading of the different compartments in the knee [8]. Thus, it offers great benefits for the assessment of patients after realignment procedures such as medial opening wedge high tibial osteotomy (OWHTO). Additionally, it allows an early detection of osteoarthritis.

Hirschmann et al. have shown how useful the combined assessment of alignment data and tracer uptake intensity distribution is in patients after total knee arthroplasty. Here, the remodeling of the bone with regard

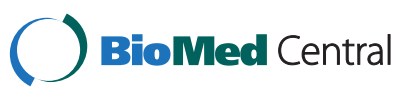


to the total knee arthroplasty position is assessed [4]. In patients after realignment surgery such as correction osteotomies, the deloading effect on the joint can be directly visualized.

However, neither a standardized algorithm for investigating patients after OWHTO nor a specific localization scheme to accurately map the examined tracer uptake volume in these patients has been published.

It was our primary purpose to introduce a novel standardized SPECT/CT algorithm including a specific localization scheme for patients undergoing high tibial osteotomy, evaluate the inter- and intra-observer reliability, and assess the clinical applicability for follow-up of patients after high tibial osteotomy. The proposed algorithm aims to improve the evaluation of patients before and after high tibial osteotomy.

\section{Methods}

Twenty-two consecutive patients (male/female 17:5, mean age $47 \pm 10$ ) with 23 knees (male/female 18:5) undergoing medial OWHTO for medial joint compartment overloading or medial tibiofemoral osteoarthritis due to mechanical varus alignment were prospectively included in this study. Exclusion criteria were posttraumatic osteoarthritis, open growth plates, corticosteroid use within the last 6 months, known history of avascular necrosis, osteochondritis dissecans, meniscal chondrocalcinosis, tumor, Paget's disease, joint infection, periarticular fracture, neuropathic arthropathy, reactive arthritis, gout, or a pregnancy.

${ }^{99 \mathrm{~m}}$ Tc-hydroxymethylene-diphosphonate (HDP)-SPECT/ CT images of these consecutive patients were prospectively collected and then evaluated. The study was approved by the local ethical committee. All procedures were performed in accordance with the ethical standards of the responsible committee and with the guidelines of the Helsinki Declaration of 1975, as revised in 2008. All patients gave written informed consent before inclusion into the study.

SPECT/CT was performed using a hybrid system (Symbia T16, Siemens, Erlangen, Germany), which is equipped with a pair of low-energy, high-resolution collimators and a dual-head gamma camera with an integrated 16-slice CT scanner (collimation of $16 \times 0.75 \mathrm{~mm}$ ). In all patients, a $500-\mathrm{MBq}{ }^{99 \mathrm{~m}} \mathrm{Tc}-\mathrm{HDP}$ tracer (Mallinckrodt, Wollerau, Switzerland) was used.

Planar scintigraphic images were taken in three phases: the perfusion phase (immediately after injection), the
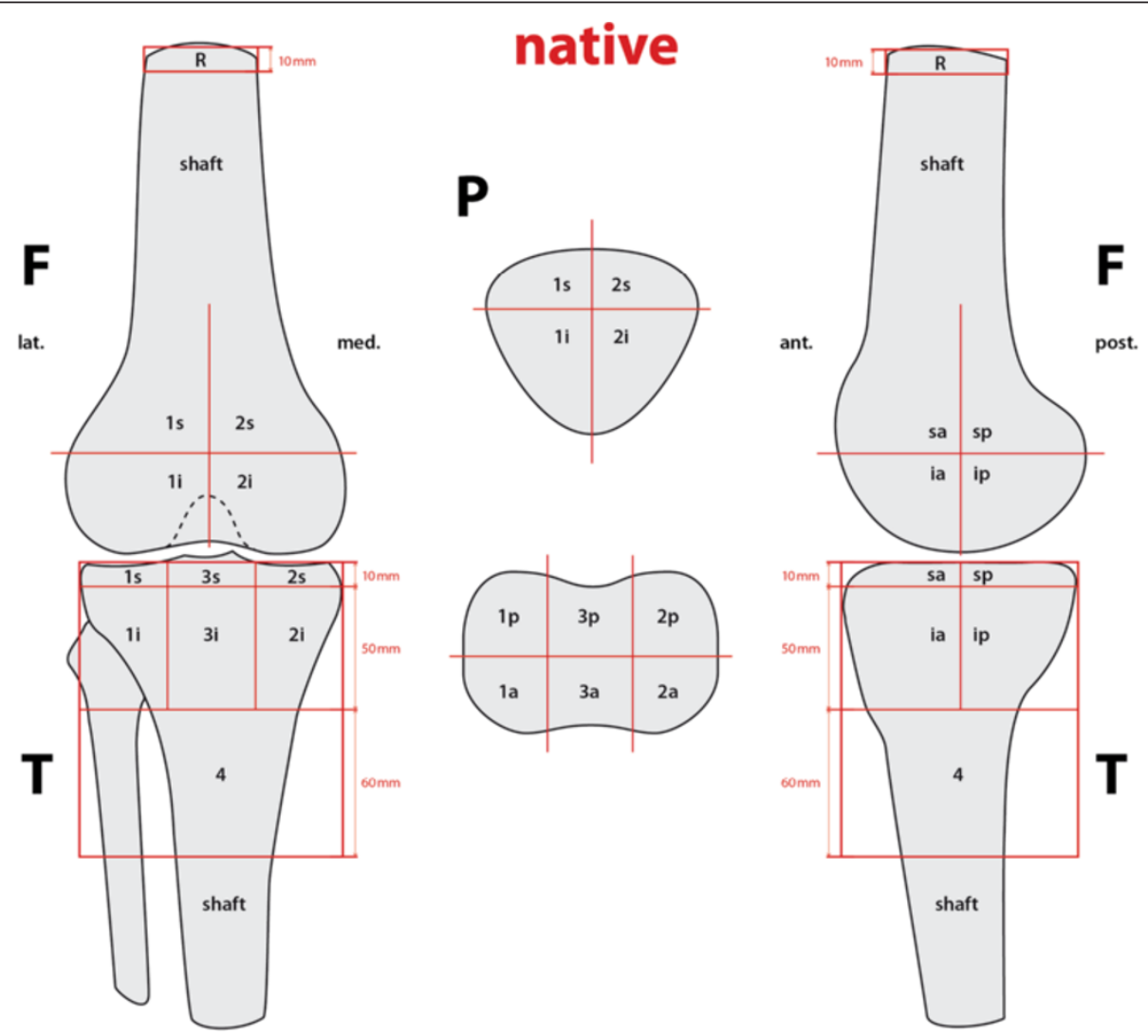

Figure 1 Mapping scheme used for localization of areas of increased SPECT/CT tracer uptake values in native knees. $F$ femur, $T$ tibia, $P$ patella, $R$ reference zone, 1 medial, 2 lateral, 3 tibial intercondylar area, 4 distal plate area, s superior, $i$ inferior, $a$ anterior, $p$ posterior. 
soft tissue phase (from 1 to 5 min after injection), and the delayed metabolic phase (from $2 \mathrm{~h}$ after injection). SPECT/CT was performed with a matrix size of $128 \times 128$, an angle step of 32, and a time per frame of $25 \mathrm{~s} 2 \mathrm{~h}$ after injection. The CT protocol was modified according to the Imperial Knee Protocol [9].

Reconstructed data was displayed in axial, coronal, and sagittal planes. SPECT/CT images were analyzed using a customized validated software (IntroSPECT®, OrthoImagingSolutions Ltd., London, UK), which is able to quantify tracer uptake volumetrically in $3 \mathrm{D}$ and anatomically localize these areas of increased tracer uptake [10].

\section{Specific tracer uptake analysis}

The proposed localization scheme defines 9 femoral, 8 patellar, and 13 tibial zones to accurately map the examined tracer uptake volume in each anatomical area of interest. In order to have comparable volumes, we used an identical localization scheme adjusted to both native (Figure 1) and operated (Figure 2) knees. Maximum values (mean \pm standard deviation, median, and range) for each area of the localization scheme were recorded as well as normalized values for the intensity of SPECT/CT tracer uptake calculated. For normalization, a specific area within the femoral shaft was used as the reference region, as previously published [10]. Thus, obtained values were dimensionless and comparable.

Pre- and postoperative mechanical alignment on SPECT/CT is explained in Figure 3. It was assessed on SPECT/CT using a custom-made previously validated software (Orthoexpert v1.15@), OrthoImagingSolutions Ltd., London, UK) [11].

All measurements were done twice by two independent observers with a 2-week interval between analyses. Both observers were blinded to results from previous observations.

\section{Statistical analysis}

Data were analyzed using SPSS 17.0 (SPSS, Chicago, IL, USA). Sample size was calculated according to the reported estimates for reliability studies using intra-class correlation coefficients (ICCs) [12].

The inter- and intra-observer reliability of the localization scheme, grading of the tracer activity, and mechanical

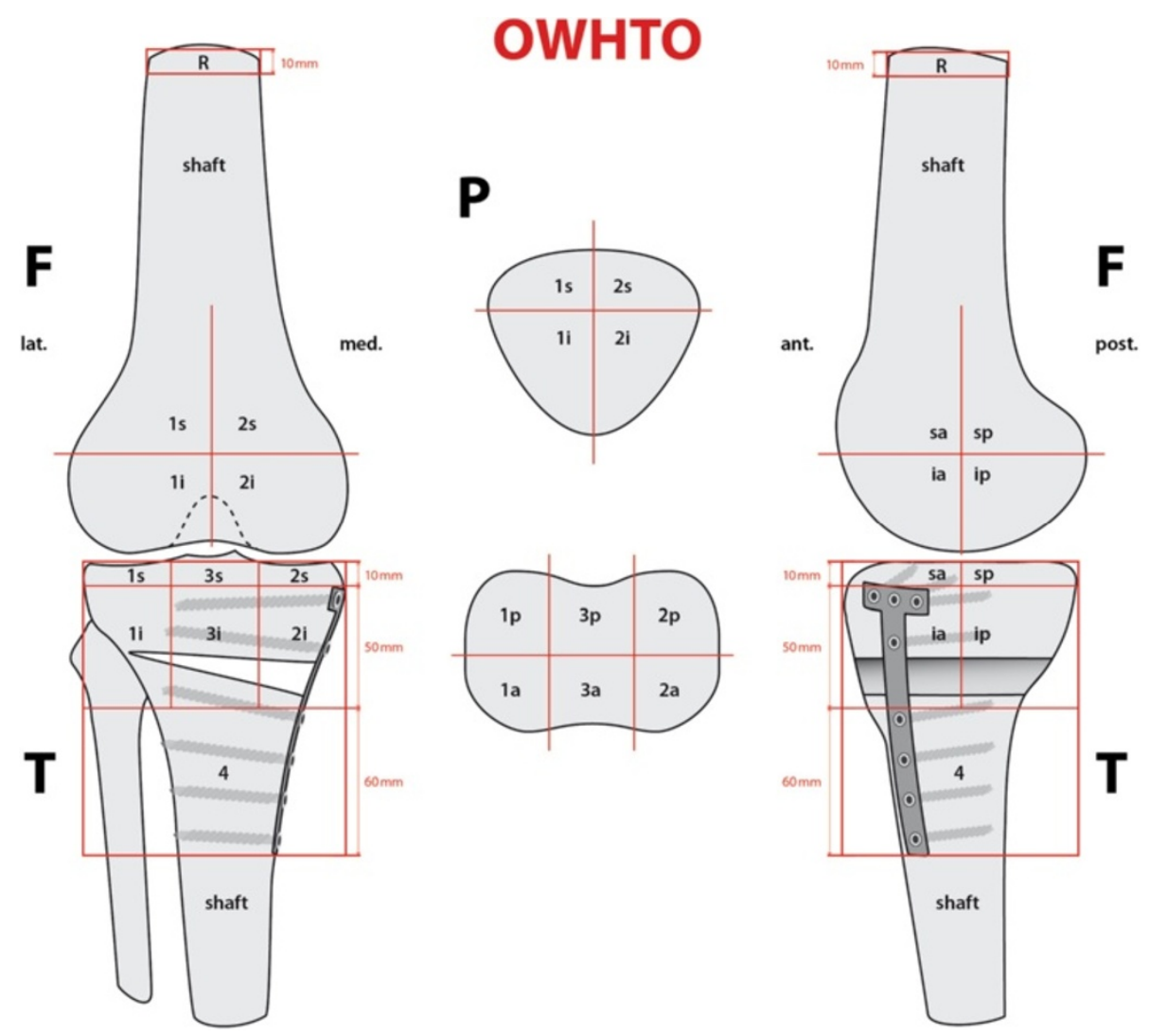

Figure 2 Mapping scheme used for localization of areas of increased SPECT/CT tracer uptake values in knees after OWHTO. $F$ femur, $T$ tibia, $P$ patella, $R$ reference zone, 1 medial, 2 lateral, 3 tibial intercondylar area, 4 distal plate area, $s$ superior, $i$ inferior, $a$ anterior, $p$ posterior. 


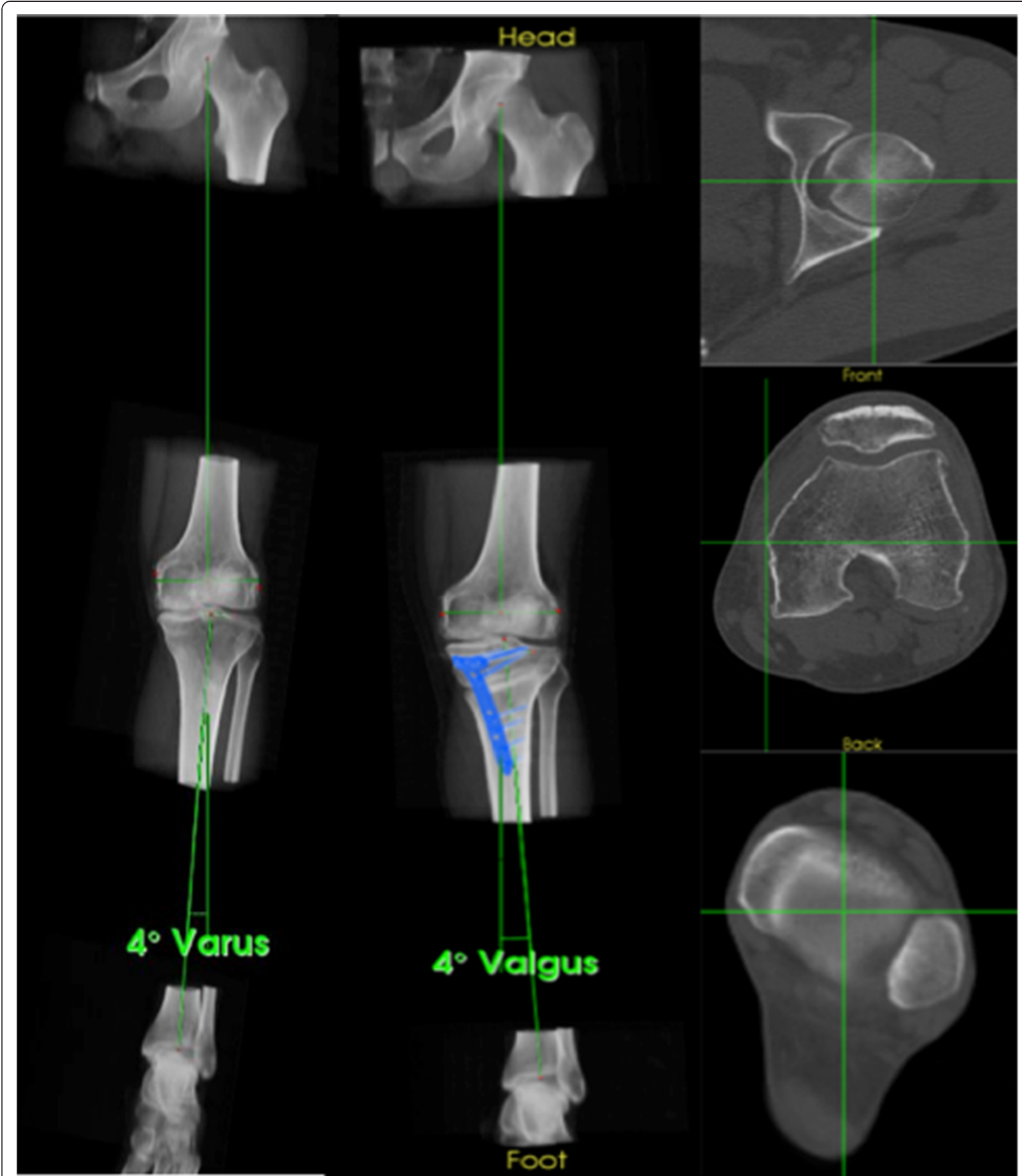

Figure 3 Pre- and postoperative mechanical alignment on SPECT/CT. The lower limb is aligned using the mechanical axis determined by the femoral head center, the medial and lateral femoral epicondyles, the center of the tibial plateau, and the talar center. Here, a pre- (4 $4^{\circ}$ arus) and a postoperative ( $4^{\circ}$ Valgus) knee are shown.

alignment measurements were determined by calculating the ICC. An ICC value of 1 indicates perfect reliability, $0.81-1$ very good reliability, and $0.61-0.80$ good reliability [12]. The inter- and intra-observer variabilities in mechanical alignment measurements were also calculated. 


\section{Results}

The localization scheme proved to be useful and easily applicable in all 23 knees. The scheme defined the relevant areas, and the ${ }^{99 \mathrm{~m}} \mathrm{Tc}-\mathrm{HDP}$ tracer uptake could be located to specific anatomical regions in all cases. Mean ICC for inter- and intra-observer reliability (OR) for all regions (femur, tibia, and patella) and both schemes ranked around 0.99 and 0.97 , respectively. Inter- and intra-OR for pre- and postoperative adjusted schemes were calculated separately, showing high ICCs for both schemes. The ICCs are presented in Tables 1 and 2 . Figure 4 shows the application of the localization scheme in a typical patient.

SPECT/CT images were of sufficient quality to determine the necessary anatomical landmarks (Figure 5). When comparing the obtained measurements for alignment, a strong agreement between the two observers

\begin{tabular}{|c|c|c|c|c|}
\hline \multirow{2}{*}{ Location } & \multirow[t]{2}{*}{ Type } & \multicolumn{2}{|c|}{ Intra-observer reliability } & \multirow{2}{*}{$\begin{array}{l}\text { Inter-observer reliability } \\
\text { Observer 1-Observer } 2\end{array}$} \\
\hline & & Observer 1 & Observer 2 & \\
\hline F1sa & Ratio & 0.995 & 0.958 & 0.988 \\
\hline F2sa & Ratio & 0.959 & 0.992 & 0.989 \\
\hline F1sp & Ratio & 1 & 1 & 1 \\
\hline $\mathrm{F} 2 \mathrm{sp}$ & Ratio & 0.999 & 0.999 & 1 \\
\hline F1ia & Ratio & 0.985 & 0.953 & 0.988 \\
\hline F2ia & Ratio & 0.983 & 0.97 & 0.988 \\
\hline F1ip & Ratio & 0.989 & 0.983 & 0.999 \\
\hline F2ip & Ratio & 0.997 & 0.994 & 0.998 \\
\hline P1s & Ratio & 0.996 & 0.996 & 0.998 \\
\hline P2s & Ratio & 0.996 & 0.986 & 0.996 \\
\hline P1i & Ratio & 0.944 & 0.985 & 0.982 \\
\hline$P 2 i$ & Ratio & 0.907 & 0.942 & 0.985 \\
\hline T1sa & Ratio & 0.981 & 0.984 & 0.997 \\
\hline T3sa & Ratio & 0.993 & 0.99 & 0.998 \\
\hline T2sa & Ratio & 0.947 & 0.975 & 0.984 \\
\hline T1sp & Ratio & 0.993 & 0.987 & 0.997 \\
\hline T3sp & Ratio & 0.977 & 0.98 & 0.989 \\
\hline T2sp & Ratio & 0.993 & 0.991 & 0.996 \\
\hline T1ia & Ratio & 0.984 & 0.964 & 0.99 \\
\hline T3ia & Ratio & 0.982 & 0.97 & 0.989 \\
\hline T2ia & Ratio & 0.898 & 0.975 & 0.977 \\
\hline T1ip & Ratio & 0.997 & 0.937 & 0.985 \\
\hline T3ip & Ratio & 0.982 & 0.98 & 0.988 \\
\hline T2ip & Ratio & 0.975 & 0.994 & 0.993 \\
\hline T4 & Ratio & 0.969 & 0.999 & 0.993 \\
\hline Total & Ratio & 0.977 & 0.979 & 0.991 \\
\hline
\end{tabular}

Table 2 Inter- and intra-OR (ICC) of ${ }^{99 \mathrm{~m}} \mathrm{Tc}$-HPD-SPECT/CT activity using the postoperative localization scheme for all regions

\begin{tabular}{|c|c|c|c|c|}
\hline \multirow[t]{2}{*}{ Location } & \multirow[t]{2}{*}{ Type } & \multicolumn{2}{|c|}{ Intra-observer reliability } & \multirow{2}{*}{$\begin{array}{l}\text { Inter-observer reliability } \\
\text { Observer } 1 \text {-Observer } 2\end{array}$} \\
\hline & & Observer 1 & Observer 2 & \\
\hline F1sa & Ratio & 0.991 & 0.976 & 0.97 \\
\hline F2sa & Ratio & 0.96 & 0.97 & 0.999 \\
\hline F1sp & Ratio & 0.94 & 0.999 & 0.994 \\
\hline $\mathrm{F} 2 \mathrm{sp}$ & Ratio & 0.779 & 0.988 & 0.986 \\
\hline F1ia & Ratio & 0.993 & 0.992 & 0.982 \\
\hline F2ia & Ratio & 0.979 & 0.92 & 0.948 \\
\hline F1ip & Ratio & 0.983 & 0.975 & 0.996 \\
\hline F2ip & Ratio & 0.958 & 0.965 & 0.974 \\
\hline P1s & Ratio & 0.997 & 0.987 & 0.991 \\
\hline P2s & Ratio & 0.965 & 0.976 & 0.983 \\
\hline P1i & Ratio & 0.971 & 0.989 & 0.991 \\
\hline P2i & Ratio & 0.974 & 0.983 & 0.987 \\
\hline T1sa & Ratio & 0.958 & 0.925 & 0.979 \\
\hline T3sa & Ratio & 0.979 & 0.989 & 0.965 \\
\hline T2sa & Ratio & 0.972 & 0.963 & 0.984 \\
\hline T1sp & Ratio & 0.973 & 0.983 & 0.992 \\
\hline T3sp & Ratio & 0.997 & 0.992 & 0.99 \\
\hline T2sp & Ratio & 0.956 & 0.986 & 0.995 \\
\hline T1ia & Ratio & 0.983 & 0.985 & 0.994 \\
\hline T3ia & Ratio & 0.988 & 0.987 & 0.983 \\
\hline T2ia & Ratio & 0.963 & 0.929 & 0.997 \\
\hline T1ip & Ratio & 0.995 & 0.998 & 0.994 \\
\hline T3ip & Ratio & 0.983 & 0.996 & 0.968 \\
\hline T2ip & Ratio & 0.999 & 0.736 & 0.999 \\
\hline T4 & Ratio & 0.998 & 0.992 & 0.999 \\
\hline Total & Ratio & 0.969 & 0.967 & 0.933 \\
\hline
\end{tabular}

was found: overall mean ICCs were 0.99 (inter-OR) and 0.98 (intra-OR). The median differences (ranges) in mechanical alignment measurements before and after osteotomy between the two observers (inter-observer) and within the measurements of the first observer (intra-observer) are shown in Table 3.

\section{Discussion}

Despite the paucity of yet available literature on the use of SPECT/CT in orthopedic patients, several promising applications of this imaging modality have been reported $[2,4,6,8,10,13,14]$. The purpose of this study was to evaluate the applicability of a SPECT/CT localization scheme and its potential benefits in patients before and after HTO.

The most important findings of this study were as follows: The proposed algorithm for investigating patients 


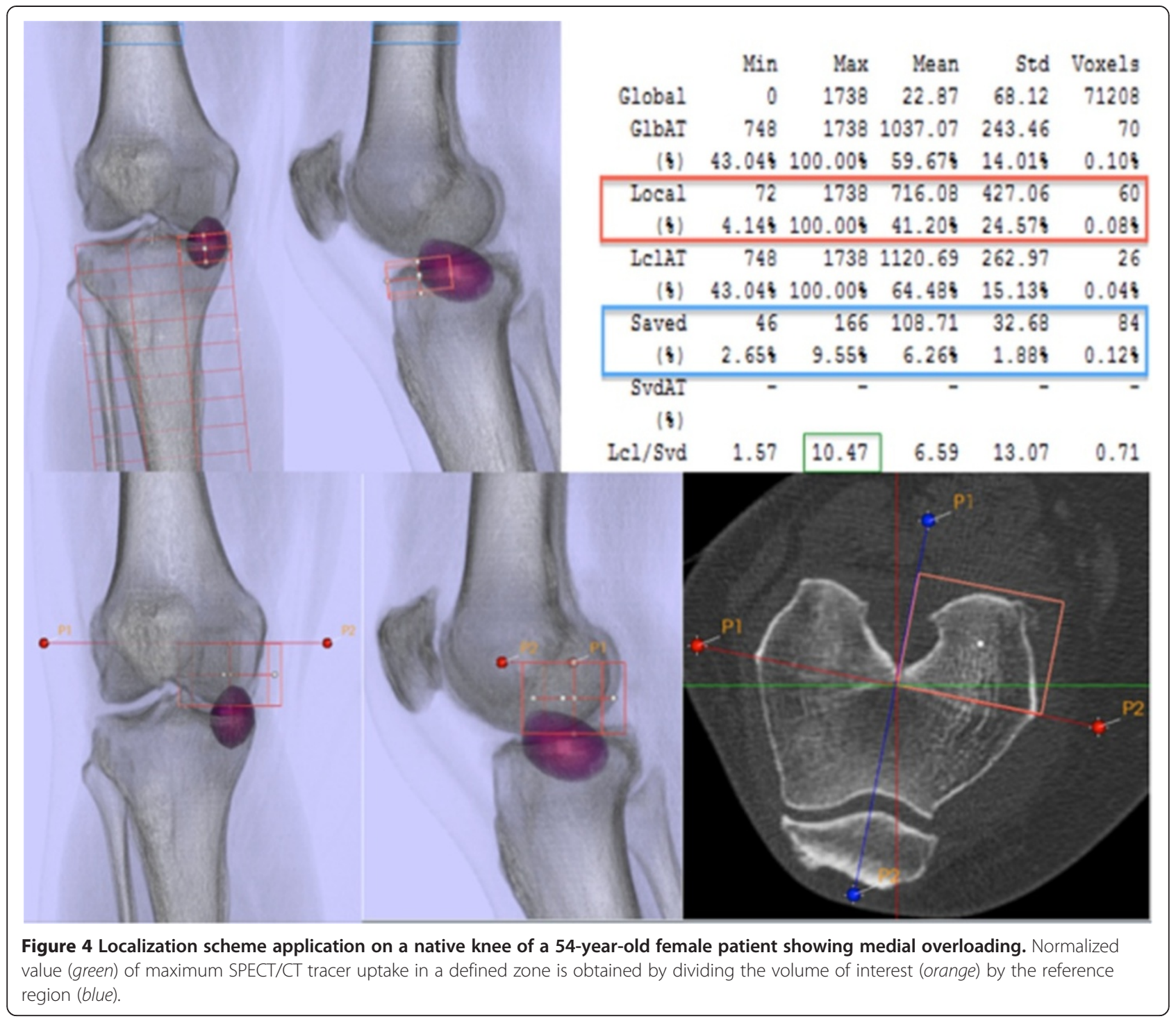

before and after OWHTO using a specific localization scheme to accurately allocate the SPECT/CT tracer uptake proved to be clinically feasible in all patients showing near-perfect inter- and intra-OR. This is the first study to describe SPECT/CT tracer uptake in knees after HTO.

The described method of assessment of the mechanical leg axis is easily applicable and also showed high inter- and intra-OR. Well-defined and reliable bony landmarks such as center of the femoral head, anatomical epicondylar line, center of the tibial plateau, and talar center were used as reference points for determination of alignment $[9,15,16]$.

For effective unloading of the medial knee compartment, Fujisawa et al. [17] recommended to correct the weight bearing line to a point $62 \%$ laterally on the tibial plateau diameter. To date, the degree of alignment correction by $\mathrm{HTO}$ is mainly based on the findings of
Fujisawa et al. with only minor adjustments in recent years [18]. However, the question of which optimal correction should be achieved in every patient remains unanswered. Due to its unique visualization of the HTO unloading effect, the proposed algorithm might be helpful in establishing an individualized degree of correction and deciding whether the operated knee was adequately, over-, or undercorrected. Clearly, it can be expected that a preoperatively increased bone tracer uptake in the medial compartment will be reduced after a wellperformed HTO, a finding which can be confirmed from our data here. In addition, we have proven in a previous study that bone tracer uptake in SPECT/CT reflects the loading pattern of the knee joint [8].

Since diphosphonate-based tracer $\left({ }^{99 \mathrm{~m}} \mathrm{Tc}-\mathrm{HDP}\right.$ and $\left.{ }^{99 \mathrm{~m}} \mathrm{Tc}-\mathrm{MDP}\right)$ uptake is known to correlate with osteoarthritic joint changes and with knee overloading $[8,19,20]$, SPECT/CT can be considered as a valuable diagnostic 


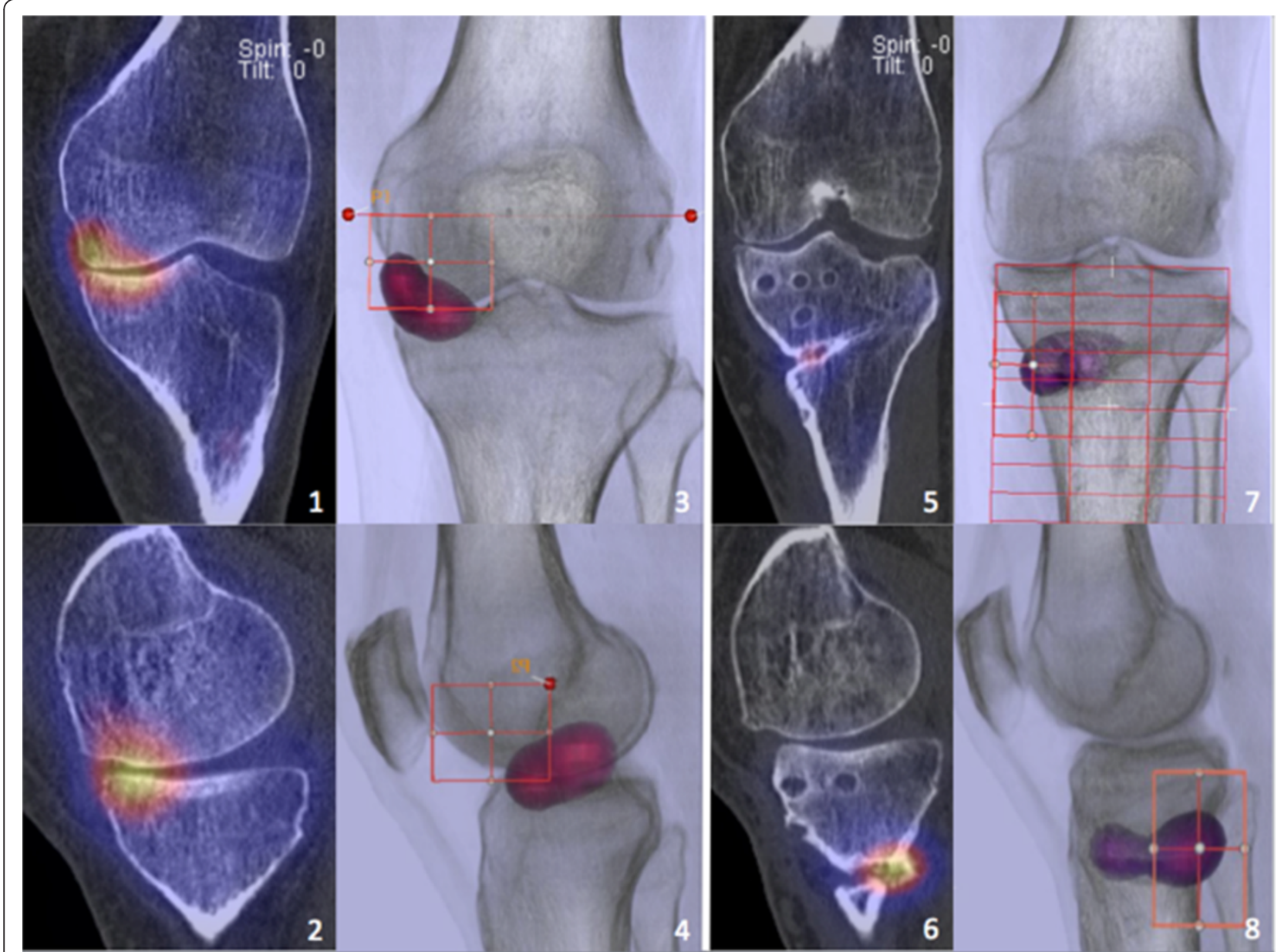

Figure 5 SPECT/CT images and 3D reconstructions before and after OWHTO. Left: SPECT/CT images $(1,2)$ and 3D radiolucent reconstructions $(3,4)$ of a 55-year-old patient before OWHTO showing medial compartment overloading. Right: 1-year postoperative SPECT/CT images $(5,6)$ and $3 \mathrm{D}$ reconstructions $(7,8)$ reveal an unloading effect of the surgically achieved correction and an advancing consolidation of the osteotomy gap with physiological increased osseous metabolism.

tool for an early detection of patients who considerably benefit from a timely realignment treatment such as HTO.

Due to the present study, SPECT/CT should be used for follow-up of patients before and after realignment surgery such as high tibial osteotomy or patellofemoral deloading.

However, the use of SPECT/CT should still be carefully indicated as the radiation burden to the patient equals three to four times that of a long leg radiograph. Aiming for a further reduction of the radiation dose, we

Table 3 Median differences (ranges) of pre- and postoperative mechanical alignment for intra- and inter-observer testing

\begin{tabular}{lcc}
\hline & Preoperative & Postoperative \\
\hline Inter-observer variation $(\mathrm{deg})$ & $1(0-2)$ & $1(0-2)$ \\
Intra-observer variation $(\mathrm{deg})$ & $1(0-1)$ & $0(0-2)$ \\
\hline
\end{tabular}

introduced a specific low-dose SPECT/CT protocol [9]. Moreover, SPECT/CT may substitute lengthy series of less conclusive imaging modalities such as long leg radiographs and thereby achieve a reduction of the overall radiation burden.

Current SPECT image resolution is still considerably lower $(4 \mathrm{~mm})$ than CT $(0.7 \mathrm{~mm})$. This mismatch does lead to some inaccuracy of SPECT localization. However, in future SPECT/CT systems, this will be resolved.

\section{Conclusions}

In summary, the presented SPECT/CT algorithm is highly reliable and clinically feasible. Evaluation of patients before and after OWHTO using SPECT/CT with regard to the mechanical leg alignment provides the surgeon with helpful additional information about the loading history of the knee joint. The clinical utility of this proposed algorithm and its localization scheme should be further investigated in clinical studies. 


\section{Competing interests}

The authors declare that they have no competing interests.

\section{Authors' contributions}

$A M, M D, E T, H R$, and MTH participated in the design of the study, performed the data analysis, and drafted the manuscript. All authors read and approved the final manuscript.

\section{Acknowledgements}

We greatly thank Deutsche Arthrose Hilfe e.V., Germany, for the financial support of the study and Jürg Schmutz for his illustrative work explaining the algorithm.

\section{Author details}

'Department of Orthopaedic Surgery and Traumatology, Kantonsspital Baselland-Bruderholz, Bruderholz 4101, Switzerland. ${ }^{2}$ Institute for Radiology and Nuclear Medicine, Kantonsspital Baselland-Bruderholz, Bruderholz 4101, Switzerland.

Received: 1 July 2013 Accepted: 3 December 2013

Published: 10 December 2013

\section{References}

1. Filippi L, Schillaci O: Usefulness of hybrid SPECT/CT in 99mTc-HMPAOlabeled leukocyte scintigraphy for bone and joint infections. J NuCl Med 2006, 47(12):1908-1913.

2. Hirschmann MT, Davda K, Iranpour F, Rasch H, Friederich NF: Combined single photon emission computerised tomography and conventional computerised tomography (SPECT/CT) in patellofemoral disorders: a clinical review. Int Orthop 2011, 35(5):675-680.

3. Hirschmann MT, Iranpour F, Konala P, Kerner A, Rasch H, Cobb JP, Friederich NF: A novel standardized algorithm for evaluating patients with painful total knee arthroplasty using combined single photon emission tomography and conventional computerized tomography. Knee Surg Sports Traumatol Arthrosc 2010, 18(7):939-944.

4. Hirschmann MT, Konala P, Iranpour F, Kerner A, Rasch H, Friederich NF: Clinical value of SPECT/CT for evaluation of patients with painful knees after total knee arthroplasty- a new dimension of diagnostics? BMC Musculoskelet Disord 2011, 12:36.

5. Hirschmann MT, Mathis D, Afifi FK, Rasch H, Henckel J, Amsler F, Wagner CR, Friederich NF, Arnold MP: Single photon emission computerized tomography and conventional computerized tomography (SPECT/CT) for evaluation of patients after anterior cruciate ligament reconstruction: a novel standardized algorithm combining mechanical and metabolic information. Knee Surg Sports Traumatol Arthrosc 2012, 21:965-974.

6. Knupp M, Pagenstert Gl, Barg A, Bolliger L, Easley ME, Hintermann B: SPECT-CT compared with conventional imaging modalities for the assessment of the varus and valgus malaligned hindfoot. J Orthop Res 2009, 27(11):1461-1466.

7. Konala P, Iranpour F, Kerner A, Rasch H, Friederich NF, Hirschmann MT: Clinical benefit of SPECT/CT for follow-up of surgical treatment of osteochondritis dissecans. Ann Nucl Med 2010, 24(8):621-624.

8. Hirschmann MT, Schon S, Afifi FK, Amsler F, Rasch H, Friederich NF, Arnold MP: Assessment of loading history of compartments in the knee using bone SPECT/CT: a study combining alignment and 99mTc-HDP tracer uptake/ distribution patterns. J Orthop Res 2013, 31(2):268-274.

9. Henckel J, Richards R, Lozhkin K, Harris S, Baena FM R y, Barrett AR, Cobb JP: Very low-dose computed tomography for planning and outcome measurement in knee replacement. The imperial knee protocol. J Bone Joint Surg Br 2006, 88(11):1513-1518.

10. Hirschmann MT, Wagner CR, Rasch H, Henckel J: Standardized volumetric 3D-analysis of SPECT/CT imaging in orthopaedics: overcoming the limitations of qualitative 2D analysis. BMC Med Imaging 2012, 12:5.

11. Rasch H, Falkowski AL, Forrer F, Henckel J, Hirschmann MT: 4D-SPECT/CT in orthopaedics: a new method of combined quantitative volumetric 3D analysis of SPECT/CT tracer uptake and component position measurements in patients after total knee arthroplasty. Skeletal Radiol 2013, 42(9):1215-1223.

12. Walter SD, Eliasziw M, Donner A: Sample size and optimal designs for reliability studies. Stat Med 1998, 17(1):101-110.
13. Hirschmann MT, Mathis D, Rasch H, Amsler F, Friederich NF, Arnold MP: SPECT/CT tracer uptake is influenced by tunnel orientation and position of the femoral and tibial ACL graft insertion site. Int Orthop 2013, 37(2):301-309.

14. Hirschmann MT, Iranpour F, Davda K, Rasch H, Hugli R, Friederich NF: Combined single-photon emission computerized tomography and conventional computerized tomography (SPECT/CT): clinical value for the knee surgeons? Knee Surg Sports Traumatol Arthrosc 2010, 18(3):341-345.

15. Victor J, Van Doninck D, Labey L, Innocenti B, Parizel PM, Bellemans J: How precise can bony landmarks be determined on a CT scan of the knee? Knee 2009, 16(5):358-365.

16. Chauhan SK, Clark GW, Lloyd S, Scott RG, Breidahl W, Sikorski JM: Computer-assisted total knee replacement. A controlled cadaver study using a multi-parameter quantitative $\mathrm{CT}$ assessment of alignment (the Perth CT Protocol). J Bone Joint Surg Br 2004, 86(6):818-823.

17. Fujisawa Y, Masuhara K, Shiomi S: The effect of high tibial osteotomy on osteoarthritis of the knee. An arthroscopic study of 54 knee joints. Orthop Clin North Am 1979, 10(3):585-608.

18. Agneskirchner JD, Hurschler C, Wrann CD, Lobenhoffer P: The effects of valgus medial opening wedge high tibial osteotomy on articular cartilage pressure of the knee: a biomechanical study. Arthroscopy 2007, 23(8):852-861

19. Siegel Y, Golan H, Thein R: 99mTc-methylene diphosphonate single photon emission tomography of the knees: intensity of uptake and its correlation with arthroscopic findings. Nucl Med Commun 2006, 27(9):689-693.

20. Kraus VB, McDaniel G, Worrell TW, Feng S, Vail TP, Varju G, Coleman RE: Association of bone scintigraphic abnormalities with knee malalignment and pain. Ann Rheum Dis 2009, 68(11):1673-1679.

doi:10.1186/1749-799X-8-46

Cite this article as: Mucha et al:: Assessment of the loading history of patients after high tibial osteotomy using SPECT/CT - a new diagnostic tool and algorithm. Journal of Orthopaedic Surgery and Research 2013 8:46.

\section{Submit your next manuscript to BioMed Central and take full advantage of:}

- Convenient online submission

- Thorough peer review

- No space constraints or color figure charges

- Immediate publication on acceptance

- Inclusion in PubMed, CAS, Scopus and Google Scholar

- Research which is freely available for redistribution

Submit your manuscript at www.biomedcentral.com/submit
C BioMed Central 